Grasp i nput opt i mizat i on taki ng cont act posi ti on and obj ect i nf or mat i on uncertai nt i es i nt o consi der at i on

\begin{tabular}{|c|c|}
\hline  & Fungt ammasan Papat, Wat anabe Tet suy ou \\
\hline $\begin{array}{l}\text { j our nal or } \\
\text { publ i cat i on ti tl e }\end{array}$ & I EEE Transacti ons on robotics \\
\hline vol une & 28 \\
\hline nunber & 5 \\
\hline page $r$ ange & $1170-1177$ \\
\hline year & 2012-01- 01 \\
\hline URL & ht t p: //hdl . handl e. net /2297/35244 \\
\hline
\end{tabular}




\title{
Grasp input optimization taking contact position and object information uncertainties into consideration
}

\author{
Papat Fungtammasan, and Tetsuyou Watanabe, Member, IEEE
}

\begin{abstract}
This paper presents a novel approach for grasp optimization considering contact position and object information uncertainties. In practice, it is hard to grasp an object at the designated or planned contact positions, as errors in measurement, estimation, and control usually exist. Therefore, we first formulate the influences of contact uncertainties on joint torques, contact wrenches, and frictional condition. We then include external wrench uncertainties in the required external wrenches set (REWS). Based on this formulation, we define the linear grasp optimization problem for two kinds of frictional contact models-frictional point contact (FPC) and soft finger contact-so that we can successfully in grasp an object even if deviations in contact point, object weight, and center of mass $(\mathrm{CM})$ occur. The validity of our approach is shown by means of numerical examples and the result of experiments.
\end{abstract}

\section{INTRODUCTION}

$\mathrm{G}^{\mathrm{s}}$ RASPING plays an important part in the area of robotics, especially in industrial and household robotics. Many researchers have tried to develop methods to overcome the challenges currently associated with this functionality. However, due to the complexity of the challenges, most of the grasping methods developed so far are for use only in well-defined situations [1-5]. For example, in situations where the robot can grasp the target object precisely at the designated positions, the CM can be accurately estimated, and the object is a rigid body.

In practical situations, these assumptions are virtually impossible. For various reasons, differences between the planned (or estimated) and actual values always occur (e.g., measurement, control, and modeling errors). A controller can be used to compensate for these errors, but there is no guarantee that the grasping action will be successful. Several researchers are presently tackling this problem and have proposed various methods for dealing with uncertainty in grasping. Chaeah et al. [5] represented all the uncertainties in terms of Jacobian uncertainty and proposed an adaptive PD controller for dealing with the problem. Schlegl and Buss [6] proposed hybrid closed-loop control for dealing with errors due to control and measurement. Bone and Du [7] presented a new metric that can measure the sensitivity of grasp to contact uncertainty. Zheng and Qian [8] derived the conditions for force closure grasps under contact uncertainty based on the analysis of object motions for force closure. Christopoulos

This work was supported by KAKENHI(20760162)

P. Fungtammasan and T. Watanabe are with College of Science and Engineering, Kanazawa University, Kanazawa, 920-1192, Japan and te-watanabe@ieee.org and Schrater [9] created an algorithm to find contact positions for grasping $2 \mathrm{D}$ objects with two contact points under contact uncertainty. Glover et al. [10] proposed an algorithm for generating probabilistic models of object geometry. Bereson et al. [11] described how to use task space regions to deal with uncertainty and create grasp planners. However, the approaches cited deal with uncertainties that occur before grasping takes place, in order to plan a grasp enumerating where grasping points should be and other parameters. Very few of them mention how to deal with the effects of the uncertainties that appear and have to be dealt with after the grasping action occurs.

With this in mind, we propose a new grasp optimization algorithm relevant to contact position and external wrench uncertainties. This algorithm optimizes the internal contact wrenches for grasping the object so that a robot can grasp an object even if grasping is disturbed after/during the grasping action due to these uncertainties. In the optimization, the uncertainties on frictional condition, torque limitation, and controller are considered. The effect of contact uncertainty is derived so that we need consider only the size of the uncertainty, regardless of direction. The main contributions of this paper are as follows.

Formulation of the effect of contact position uncertainty: The key issue is how to formulate the effect of contact position uncertainty in grasp optimization. We formulate the effect of contact position uncertainty on frictional condition and joint torque limitations as linear conditions. Based on the formulation, we present linear programming for optimization, which provides the internal contact wrenches and grasp inputs (such as joint torque input) for generating the required contact wrenches.

Dealing with the uncertainties of the external wrench: In practice, it is hard to estimate the exact external wrench (before grasping). However, we can estimate the maximum possible load and the range where the $\mathrm{CM}$ is likely to exist. We consider this range in the grasp optimization. Normally, external wrench uncertainty is partly affected by object information uncertainty, such as that resulting from a lack of information about the object.

Taking controller into consideration: In this paper, we propose the use of a compliance controller. This controller can compensate for external wrench, contact position uncertainty, and so on, if frictional conditions can be satisfied and the required joint torques can stay in their operational ranges. Therefore, we plan the contact wrenches so that compensation is available. We subsequently derive the desired state for the controller from the planned contact 
wrenches.

In contrast to our previous research [12], the technique presented in this paper can deal with cases in which the object grasp by the robot is oval in shape. Additionally, this paper compares the results for FPC and soft finger contact.

\section{PROBlem DEFINITION}

Contact Model: We consider two contact models: FPC, the contact wrench at contact point $C_{\mathrm{ij}}$, which consists of three dimensional force $\left(\boldsymbol{w}_{\mathrm{cij}}=\left[f_{\mathrm{x}} f_{\mathrm{y}} f_{\mathrm{z}}\right]_{\mathrm{ij}}^{\mathrm{T}}, f_{\mathrm{x}}, f_{\mathrm{y}}, f_{\mathrm{z}}\right.$ denote force component in 3 directions): and soft finger contact, the contact wrench consisting of three dimensional force and moment in the normal contact direction $\left(\boldsymbol{w}_{\mathrm{cij}}=\left[f_{\mathrm{x}} f_{\mathrm{y}} f_{\mathrm{z}} \phi\right]^{\mathrm{T}}{ }_{\mathrm{ij}}, \phi\right.$ denotes moment component)[13]. There are two models for soft finger contact constraint: the linear model and the ellipse model. However, we consider only the linear model of soft finger contact for two main reasons. First, contact wrenches that satisfy the linear model automatically satisfy the ellipse model also. Therefore the conditions formulated for the linear model (as will be seen later) can also be used for the ellipse model. Second, the complexity of the equation for the ellipse model is much higher when we consider the effect of contact uncertainty.

We assume that frictional coefficient and nominal contact positions are all given. The number of contact points does not change due to contact position perturbation.

Controller: We use a compliance controller for grasping. This can be defined as

$$
\tau=K \Delta q
$$

where $\boldsymbol{\tau}$ denotes joint torque of all joint of robot hand, $\boldsymbol{K}$ denotes diagonal controlling gain matrix, and $\Delta \boldsymbol{q}$ denotes the difference between desired and actual joint angle of all joint. Note that we assume that $\boldsymbol{K}$ is relatively large. Thus the $\Delta \boldsymbol{q}$, resulting from the compensation of external wrenches, is relatively small and so we can ignore its effect on the Jacobian matrix defined below.

Hand Posture: the nominal joint angle of the hand of the robot for grasping (grasping posture), $\boldsymbol{q}$, is assumed to be given. The corresponding nominal contact positions, $\boldsymbol{p}_{\mathrm{Cij}}$, are also given. Here, $\boldsymbol{p}_{\mathrm{Cij}}$ denotes the position for the $j^{\text {th }}$ contact point at the $i^{\text {th }}$ finger.

Object coordinate frame: The object coordinate frame is given at the estimated CM position. Normally, the CM is unknown before grasping. The distance between the estimated and actual positions is considered the CM deviation. The CM deviation causes the external moment with respect to the object frame.

Contact point uncertainty: In practice, it is very difficult for the robot to grasp an object at the planned or designated contact positions due to measurement and control errors. We regard this error as contact point uncertainty, and take it into consideration in grasp optimization. Here, we consider the range of the uncertainty regardless of direction. We denote the contact point uncertainty for $\boldsymbol{p}_{\mathrm{Cij}}$ on the object surface as
$\Delta_{\mathrm{p}} p^{\mathrm{o}}{ }_{\mathrm{Cij}}$ and uncertainty on the finger as $\Delta_{\mathrm{p}} p^{F}{ }_{\mathrm{Cij}}$. If we set the maximum values of $\Delta_{\mathrm{p}} p^{o}{ }_{\mathrm{Cij}}$ as $P_{\text {max }}^{o}$ and $\Delta_{\mathrm{p}} \boldsymbol{p}^{F_{\mathrm{Cij}}}$ as $P_{\text {max }}^{F}$, regardless of contact points, we get

$$
\left|\Delta_{p} \boldsymbol{p}_{C_{i j}}^{o}\right| \leq P_{\max }^{o},\left|\Delta_{p} \boldsymbol{p}_{C_{i j}}^{F}\right| \leq P_{\max }^{F}
$$

Required External Wrench Set (REWS) (expanded from [4]) is a set of wrenches that contains all the possible external wrenches (i.e., the estimated external wrenches and all of their uncertainties). We assume that REWS is given as a convex polyhedron and that the estimated external wrench can be presented as a convex representation

$$
\boldsymbol{w}_{e x}=\sum_{l=1}^{n_{\text {rew }}} \lambda_{l} \boldsymbol{w}_{\mathrm{vl}}, \sum_{l=1}^{n_{\text {rew }}} \lambda_{l}=1, \quad \lambda_{l} \geq 0
$$

where $\boldsymbol{w}_{\mathrm{ex}}$ denotes external wrench or given task, $\boldsymbol{w}_{\mathrm{vl}}$ denotes the $l^{\text {th }}$ vertex of REWS, $\lambda_{1}$ denotes the proportion of each vertex, and $n_{\text {rew }}$ denotes the number of the vertices.

Equation (3) indicates that if the robot can support all $\boldsymbol{w}_{\mathrm{vl}}$, then it can support $\boldsymbol{w}_{\mathrm{ex}}$ and all of its uncertainties. Therefore, we will balance the set of $\boldsymbol{w}_{\mathrm{vl}}$ instead of $\boldsymbol{w}_{\mathrm{ex}}$ in the rest of this paper.

Scenario: We consider the following situation. We make contact with an object to grasp it with a nominal grasping posture $(\boldsymbol{q})$. We apply only internal contact wrenches, which do not affect the resultant wrench to the object, since the compliance controller can compensate for the external wrench if the frictional condition is satisfied and the required joint torques stay in their operational ranges. However, there can be differences between the actual and the desired contact positions. Hence, we must apply internal contact wrenches so that we can keep grasping regardless of the contact uncertainty.

Based on the above setting, we consider the following problem.

Problem: Nominal grasping posture and the (designated) contact position are given. REWS and the coefficient of friction are also given. For this case, derive the desired internal contact wrenches for grasping the target object so that we can grasp the object even if there are contact positions and external wrench uncertainties. The optimization is under the condition that the contact wrenches must satisfy frictional conditions and the available grasp input can generate these contact wrenches.

\section{BASIC FORMULA}

In the ideal situation, the relation between external wrench, contact wrenches and joint torques can be represented by

$$
\left[\begin{array}{c}
\tau \\
-w_{v l}
\end{array}\right]=\left[\begin{array}{l}
J^{T} \\
G
\end{array}\right] w_{c}
$$

where $\boldsymbol{J}$ denotes Jacobian matrix of robot hand $\left(\boldsymbol{J}=\operatorname{diag}\left[\boldsymbol{J}_{\mathrm{i}}\right]\right.$, $\left.\boldsymbol{J}_{\mathrm{i}}=\operatorname{col}\left[\boldsymbol{J}_{\mathrm{ij}}\right]\right), \boldsymbol{G}=\operatorname{row}\left[\boldsymbol{G}_{\mathrm{ij}}\right]$ denotes grasp matrix, $\boldsymbol{w}_{\boldsymbol{c}}=\operatorname{col}\left[\boldsymbol{w}_{\boldsymbol{c} i j}\right]$ denote contact wrenches, $\operatorname{col}\left[\boldsymbol{A}_{\mathrm{i}}\right]=\left[\boldsymbol{A}^{\mathrm{T}}{ }_{1} \boldsymbol{A}^{\mathrm{T}}{ }_{2} \ldots\right]^{\mathrm{T}}, \operatorname{row}\left[\boldsymbol{A}_{\mathrm{i}}\right]=\left[\boldsymbol{A}_{1}\right.$ 
$\left.\boldsymbol{A}_{2} \ldots\right], \operatorname{diag}\left[\boldsymbol{A}_{\mathrm{i}}\right]$ denotes diagonal matrix of $\boldsymbol{A}$. The grasp matrix shows the relation between the contact wrenches and the external wrench. Note that the components $\boldsymbol{G}$ and $\boldsymbol{J}$ for the FPC and soft finger contact are different.

For the non-slip condition, $\boldsymbol{w}_{\mathrm{c}}$ must satisfy the frictional condition. The frictional constraint has been linearized into a set of inequality equations [3][14]

$$
-V w_{c} \leq 0
$$

where $\boldsymbol{V}=\operatorname{diag}\left[\boldsymbol{V}_{\mathrm{ij}}\right], \boldsymbol{V}_{\mathrm{ij}}=\operatorname{row}\left[\boldsymbol{a}_{\mathrm{lij}}\right]$ as shown in Fig.1. Equation (5) is the H-representation of the convex polyhedral friction cone at $C_{\mathrm{ij}}$. The details of $\boldsymbol{V}_{\mathrm{ij}}$, for FPC, can be found in [3]. In the case of soft finger contact, it can be obtained by converting the V-representation from [5].

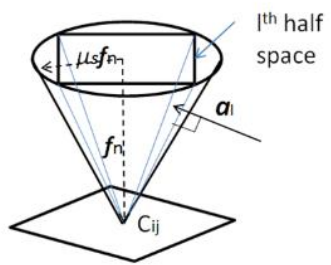

Fig. 1. Convex polyhedral friction cone for FPC at $C_{\mathrm{ij}}$.

In conventional grasp optimization, we optimize the task function $\left(\mathrm{F}_{\text {task }}\right)$ under three constraints. First, the actuator can supply enough torque. Second, all contact wrenches satisfy the frictional condition. Third, the contact wrenches can support the external wrench. The conventional grasp optimization can be shown as

$\begin{array}{ll}\min & \mathcal{F}_{\text {task }} \\ \text { subject to } & \text { 1) }\left|\boldsymbol{J}^{T} \boldsymbol{w}_{c}\right| \leq\left|\boldsymbol{\tau}_{\text {max }}\right| \\ & \text { 2) }-\boldsymbol{V} \boldsymbol{w}_{c} \leq \mathbf{0} \\ & \text { 3) } \boldsymbol{n}^{\boldsymbol{T}} \boldsymbol{w}_{c} \geq 0 \\ & \text { 4) } \boldsymbol{G} \boldsymbol{w}_{c}=-\boldsymbol{w}_{\boldsymbol{v} \boldsymbol{l}}\end{array}$

where $\tau_{\max }$ denotes maximum torque from actuator, $\boldsymbol{n}=\operatorname{col}\left[\boldsymbol{n}_{\mathrm{ij}}\right]$, $\boldsymbol{n}_{\mathrm{ij}}$ denotes unit vector of normal direction at $C_{\mathrm{ij}}$. From this linear equation, we can derive the contact wrenches that satisfy the grasp system.

In some situations, we can design the internal contact wrenches for grasping. As mentioned above, the robot can support the external wrench and its uncertainty if it can support the wrench at every vertex of REWS. Thus, from (3) and (4) we get

$$
w_{c}=-G^{+} w_{v l}+E \gamma
$$

where $\boldsymbol{G}^{+}$denotes the pseudo-inverse matrix, $\boldsymbol{E}$ denotes an orthogonal matrix, constructed from all null space of $\boldsymbol{G}$ and $\boldsymbol{\gamma}$ denotes an arbitrary vector.

The first term on the right hand side is for balancing the external wrench, while the second term represents the internal wrench, which does not affect the object motion. Consequently, if the use of a compliance controller is considered, the first term will change in accordance with the external wrench, while the second term should be applied by the robot so that the grasp can be maintained, regardless of the external wrench. In other words, the first term is determined after grasping while the second term should be determined before grasping. We will set $\gamma$ so that every $w_{c}$ can satisfy the frictional condition and its corresponding joint torques cannot exceed the maximum joint torque values. Then we can balance any external wrench contained in REWS with the constant $\gamma$.

\section{EFFECT OF CONTACT UNCERTAINTIES}

In this section, we will look at the effect of contact uncertainties on grasp optimization equation (6). First, we will discuss the physical effect when contact uncertainties occur (sections A and B). Next, we consider the effects on each constraint of grasp optimization (sections $\mathrm{C}-\mathrm{E}$ ). Finally, we summarize all the effects on the grasp optimization (section F) and demonstrate how to verify the required internal contact wrench by minimizing the applied torque.

\section{A. Contact uncertainties on object and robot hand}

Consider the contact uncertainties in Fig. 2. The robot tries to grasp the object at nominal posture (dotted line), but it actually grasps the object at a slightly deviated posture (bold line). As a result, the contact point changes from the nominal contact position (gray dot), to the deviated contact position (black dot). We have deviation on both the fingertip and object surface.



Fig. 2. The uncertainty of contact point on both finger and object at the $K^{\text {th }}$ link of $i^{\text {th }}$ finger.

With respect to contact point $C_{\mathrm{ij}}$, the relationship between the deviations of contact point $\left(p_{\text {cij }}\right)$, joint angle $\left(q_{i}\right)$, and the position of $\mathrm{CM}(\boldsymbol{r})$ are given by

$$
{ }_{p} \boldsymbol{p}_{C_{i j}}^{o}+{ }_{p} \boldsymbol{p}_{C_{i j}}^{F}=J_{i j} \boldsymbol{q}_{i}, \quad{ }_{p} \boldsymbol{p}_{C_{i j}}^{o}=G_{i j}^{T} \boldsymbol{r}
$$

\section{B. Effect on contact normal direction of contact uncertainty}

When contact uncertainty occurs, the normal contact direction will change if the contact surface is not flat. This change results from curvature at the contact point and contact point uncertainty. The relation between the deviated contact normal direction and contact position uncertainty is depicted in Fig. 3.

From Fig. 3, we can express the maximum deviated angle for the contact normal direction (i.e., the boundary), called the contact normal uncertainty, as

$$
\theta_{\max _{i j}}=2 \sin ^{-1} \frac{P_{\max }^{o}}{2 \operatorname{rad}_{i j}}
$$


where $\theta_{\text {maxij }}$ denotes maximum contact normal uncertainty at $C_{\mathrm{ij}}$, and $\operatorname{rad}_{\mathrm{ij}}$ denotes minimum curvature radius from every tangential direction at $C_{\mathrm{ij}}$, see Fig.3.

The boundary of uncertainty of the normal contact direction $\left|\Delta_{p} \boldsymbol{n}_{i j}\right|$ can be found by using the law of cosines. The maximum value of the normal direction uncertainty is

$$
\begin{aligned}
\left|\Delta_{p} \boldsymbol{n}_{i j}\right| & =\sqrt{2-2 \cos \theta} \\
\leq & \sqrt{2-2 \cos \theta_{\max }}={ }_{p} n_{\max } .
\end{aligned}
$$

where $\theta$ denotes angle between designed and actual directions, $\Delta_{\mathrm{p}} \boldsymbol{A}$ denotes uncertainty of $\boldsymbol{A}$ due to contact uncertainties. We will now use the information from equations (8)-(10) to derive the effect of contact uncertainties in the sections below.
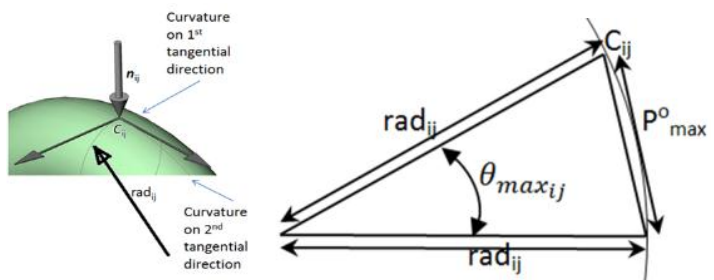

Fig. 3 Relation between maximum deviated angle $\theta_{\text {maxij }}$ and contact position uncertainty $P_{\text {max }}^{\mathrm{o}}$. Note that in this picture $\operatorname{rad}_{\mathrm{ij}}$ lie on $1^{\text {st }}$ tangential direction since the radius on this direction is the shortest.

\section{Static relationship considering contact uncertainty}

When contact uncertainties occur, the grasp matrix and contact wrenches will be affected. In order to grasp successfully, equation (4) has to be satisfied even under the effect of uncertainties. Consequently, we have

$$
-\mathbf{w}_{v l}=G w_{c}=\left(G+{ }_{p} G\right)\left(w_{c}+{ }_{p} w_{\mathbf{c}}\right)
$$

If we neglect the higher order term, we get

$$
\begin{aligned}
& { }_{p} \boldsymbol{G} \boldsymbol{w}_{\mathbf{c}}+\boldsymbol{G} \Delta_{p} \boldsymbol{w}_{\mathbf{c}}=\mathbf{0} \\
& { }_{p} \boldsymbol{w}_{\mathbf{c}}=-\boldsymbol{G}^{+}{ }_{p} \boldsymbol{G} \boldsymbol{w}_{\mathbf{c}} .
\end{aligned}
$$

As can be seen, the contact uncertainties lead to the uncertainties of contact wrenches. If we consider grasp optimization equation (6), the change in the contact wrench affects every constraint. We will consider its effect in section $\operatorname{IV}(\mathrm{D})$ and $\mathrm{IV}(\mathrm{E})$ below.

\section{Effect of contact uncertainty on the frictional condition}

From equation (11) and (12), we can see that contact uncertainty causes uncertainty in the contact wrenches. The total contact wrenches may not satisfy the frictional condition, as shown in Fig.4. Moreover, the friction cone will be disturbed if contact normal uncertainty occurs. Thus, in summary, the effect of contact uncertainty on frictional condition can be divided into two parts: the effect on the contact wrenches and the effect on the friction cone. We will show how to deal with each effect below.
In practice, it is hard to detect contact uncertainty, especially if it is small. We take this into consideration when we design or plan the constant internal wrenches for grasping (since we assume a compliance controller is used), so that frictional condition can be satisfied when we have $\Delta_{\mathrm{p}} \boldsymbol{w}_{\mathrm{c}}$.

Now we will consider $\Delta_{\mathrm{p}} \boldsymbol{w}_{\boldsymbol{c}}$, given in (12). First, we consider the effect of contact uncertainty on the components of the grasp matrix. If the object coordinate frame does not change during grasping, this effect can be expressed by

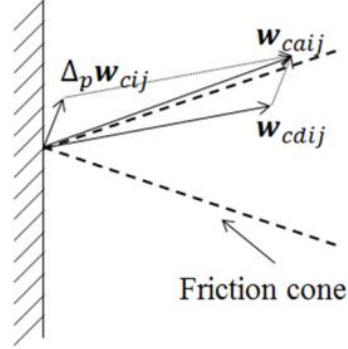

Fig. 4 The relation between nominal $\boldsymbol{w}_{\mathrm{cd}}$ and actual contact wrench $\boldsymbol{w}_{\mathrm{ca}}$

$$
\begin{aligned}
\boldsymbol{G}_{i j} & =\left[\begin{array}{c}
\mathbf{0} \\
{\left[\Delta_{p} \boldsymbol{p}_{C_{i j}}^{o} \times\right]}
\end{array}\right] \\
\boldsymbol{G}_{i j} & =\left[\begin{array}{cc}
\mathbf{0} & \mathbf{0} \\
{\left[\Delta_{p} \boldsymbol{p}_{C_{i j}}^{o} \times\right.}
\end{array}\right]
\end{aligned}
$$

From this equation, it can be seen that only the moment component of the external wrench is influenced. The uncertainty of this moment, $\Delta_{\mathrm{p}} \boldsymbol{m}=\left[\begin{array}{ll}\boldsymbol{0} & \boldsymbol{I}\end{array}\right] \Delta_{\mathrm{p}} \boldsymbol{G} \boldsymbol{w}_{\mathrm{c}}$, is expressed by

$$
\begin{aligned}
& { }_{p} \boldsymbol{m}=\sum_{i, j}\left|\Delta_{p} \boldsymbol{p}_{C_{i j}}^{o}\right|\left[\Delta_{p} \widehat{\boldsymbol{p}}_{C_{i j}}^{o} \times\right] \boldsymbol{f}_{i j} \\
& { }_{p} \boldsymbol{m}=\sum_{i, j}\left|\Delta_{p} \boldsymbol{p}_{C_{i j}}^{o}\right|\left[\Delta_{p} \widehat{\boldsymbol{p}}_{C_{i j}}^{o} \times\right] \boldsymbol{f}_{i j}+\boldsymbol{n}_{i j} \varphi_{i j}
\end{aligned}
$$

where $\boldsymbol{f}_{\mathrm{ij}}=\left[f_{\mathrm{x}} f_{\mathrm{y}} f_{\mathrm{z}}\right]_{\mathrm{ij}}^{\mathrm{T}}, \widehat{\boldsymbol{p}}_{C_{i j}}^{o}$ denotes unit vector of direction of $\boldsymbol{p}_{C_{i j}}^{o}$. First, let us consider the moment uncertainty related to $f_{\mathrm{ij}}$.

$$
\sum_{i, j}\left|\Delta_{p} \boldsymbol{p}_{C_{i j}}^{o}\right|\left|\left[\Delta_{p} \widehat{\boldsymbol{p}}_{C_{i j}}^{o} \times\right] \boldsymbol{f}_{i j}\right| \leq P_{\max }^{o} \sum_{i, j}\left|\boldsymbol{f}_{i j}\right|
$$

For FPC we can get

$$
\left|\boldsymbol{f}_{i j}\right| \leq \sqrt{1+\mu_{s i j}^{2}} n_{w_{i j}}
$$

where $\boldsymbol{n}_{\text {wij }}$ denotes the normal contact force component at $C_{\mathrm{ij}}$, $\mu_{\mathrm{sij}}$ denote original static coefficient of friction at $C_{i j}$. In the case of soft finger contact, the frictional constraint of the linear model can be shown as

$$
\left|\boldsymbol{T}_{i j} \boldsymbol{f}_{i j}\right| \leq \mu_{s_{i j}} n_{w i j}-\frac{\mu_{s_{i j}}}{\mu_{m_{i j}}}\left|\phi_{i j}\right|
$$

where $\mu_{\text {mij }}$ denote original moment coefficient of friction at $C_{i j},\left|\boldsymbol{T}_{i j} \boldsymbol{f}_{i j}\right|$ denotes magnitude of tangential contact force at 


$$
\begin{aligned}
& C_{i j} \text {. Since }\left|\boldsymbol{f}_{i j}\right| \leq\left|\boldsymbol{T}_{i j} \boldsymbol{f}_{i j}\right|+n_{\mathrm{wij}}, \text { then } \\
& \quad\left|\boldsymbol{f}_{i j}\right| \leq\left(\mu_{s i j}+1\right) n_{w_{i j}}-\frac{\mu_{s i j}}{\mu_{m i j}}\left|\phi_{i j}\right| .
\end{aligned}
$$

From (12), the boundary of the contact wrench uncertainty becomes,

$$
\begin{aligned}
& \left|\Delta_{p} \boldsymbol{w}_{c i j}\right| \leq\left|\boldsymbol{\Xi}_{i j} \boldsymbol{G}^{+}\left[\begin{array}{l}
\mathbf{0} \\
\boldsymbol{I}
\end{array}\right]\right|\left|\Delta_{p} \boldsymbol{m}\right| \\
& \left|\Delta_{p} \boldsymbol{w}_{c i j}\right| \leq\left|\boldsymbol{\Xi}_{i j} \boldsymbol{G}^{+}\left[\begin{array}{cc}
\boldsymbol{O} & \boldsymbol{O} \\
\boldsymbol{I} & \boldsymbol{n}_{\boldsymbol{i j}}
\end{array}\right]\right|\left|\Delta_{p} \boldsymbol{m}\right| \quad \text { (soft) }
\end{aligned}
$$

where $\boldsymbol{\Xi}_{i j}$ denotes separation matrix for $C_{\mathrm{ij}}\left(\boldsymbol{w}_{\mathbf{c i j}}=\boldsymbol{\Xi}_{i j} \boldsymbol{w}_{\mathbf{c}}\right)$. The magnitude of each contact wrench uncertainty is bounded

$$
\left|\Delta_{p} \boldsymbol{w}_{c i j}\right| \leq{ }_{p} w_{c i j_{\max }} \boldsymbol{n}_{\boldsymbol{\mu}}^{T} \boldsymbol{w}_{\boldsymbol{c}}
$$

where

$$
\begin{aligned}
& { }_{p} w_{c i j_{\max }}=\left|\mathbf{\Xi}_{i j} \boldsymbol{G}^{+}\left[\begin{array}{l}
\mathbf{0} \\
\mathbf{I}
\end{array}\right]\right| P_{\max }^{o} \\
& { }_{p} w_{c i j_{\max }}=\left|\boldsymbol{\Xi}_{i j} \boldsymbol{G}^{+}\left[\begin{array}{cc}
\boldsymbol{O} & \boldsymbol{O} \\
\boldsymbol{I} & \boldsymbol{n}_{\boldsymbol{i j}}
\end{array}\right]\right| P_{\max }^{o} \\
& \boldsymbol{n}_{\mu i j}^{T}=\left[\sqrt{1+\mu_{s i j}^{2}} \boldsymbol{n}_{i j}^{T}\right]
\end{aligned}
$$

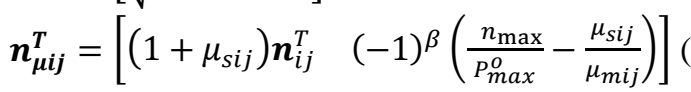

where $\boldsymbol{n}_{\mu}=\operatorname{col}\left[\boldsymbol{n}_{\mu i j}\right]$, and $\beta \in\{1,2\}$. Note that for soft finger contact, equation (18) indicates two inequality equations.

Now consider the effect of contact uncertainty on the friction cone. If $\Delta_{\mathrm{p}} \boldsymbol{n}_{\mathrm{ij}}$ (from (10)) occurs, the friction cone will deviate from the designated position and orientation. The change in the friction cone can be represented as an adjustment to the coefficient of friction. The new coefficient of friction, called the equivalent coefficient of friction, is expressed by

$$
\begin{aligned}
& \mu_{s e q_{i j}}=\tan \left(\tan ^{-1} \mu_{s i j}-\theta_{\max _{i j}}\right) \\
& \mu_{\text {meq }_{i j}}=\cos \left(\theta_{\text {max }_{i j}}\right) \mu_{m_{i j}}
\end{aligned}
$$

where $\mu_{\text {seqij }}$ and $\mu_{\text {meqij }}$ denotes equivalent static and moment coefficient of friction at $C_{\mathrm{ij}}$ respectively. Next, we linearize this friction cone and create matrix $V_{\text {eq }}$ with the $\mu_{\text {seqij }}$ and $\mu_{\text {meqij, }}$, in the same way as the derivation of (5).

In summary, we must consider the effect of contact wrench uncertainty (18) and the effect of the changing of friction cone (19). We compensate for (19) by increasing the contact force in the normal direction, and using $\boldsymbol{V}_{\mathrm{eq}}$ instead of $\boldsymbol{V}$ to compensate for the change in the friction cone. Consequently, (5) becomes

$$
-V_{e q}\left(I-\operatorname{col}\left[\Delta_{p} w_{c i j_{\max }}\right] n n_{\mu}^{T}\right) w_{c} \leq \mathbf{0} .
$$

We use (20), instead of (5), to verify the frictional condition for the grasping system with uncertainty so that the frictional condition can be satisfied even when the grasping force (unexpectedly) changes due to the deviation.

\section{E. Effect of contact uncertainty on joint torques}

We now consider the deviation of joint torques due to contact position perturbation. From (4), the $k^{\text {th }}$ joint torque of the $i^{\text {th }}$ finger becomes

$$
\begin{gathered}
\tau_{i k}+{ }_{p} \tau_{i k}=\sum_{j}\left(\left[J_{i j}^{T}\right]_{k}+\left[\Delta_{p} J_{i j}^{T}\right]_{k}\right)\left(\boldsymbol{w}_{c i j}+{ }_{p} \boldsymbol{w}_{c i j}\right) \\
=\tau_{i k}+\sum_{j}\left[\Delta_{p} \boldsymbol{J}_{i j}^{T}\right]_{k} \boldsymbol{w}_{c i j}+\sum_{j}\left[\boldsymbol{J}_{i j}^{T}\right]_{k}{ }_{p} \boldsymbol{w}_{c i j}
\end{gathered}
$$

where $[\boldsymbol{A}]_{\mathrm{k}}$ denotes the $k^{\text {th }}$ row vector of the matrix $\boldsymbol{A}$ and we ignore higher order terms. $\Sigma_{\mathrm{j}}$ indicates the summation of all contact points on $i^{\text {th }}$ finger. Since the Jacobian is a function of the joint angle and the contact point on the finger $\left(\boldsymbol{J}_{\mathrm{ij}}=J_{\mathrm{ij}}\left(q_{\mathrm{i}}, \boldsymbol{p}^{\mathrm{F}}{ }_{\mathrm{Cij}}\right)\right),\left[\Delta_{\mathrm{p}} \boldsymbol{J}_{\mathrm{ij}}\right]_{\mathrm{k}}$ can be written as

$$
\begin{aligned}
{\left[\Delta_{p} \boldsymbol{J}_{i j}^{T}\right]_{k} } & =\left[\left[\Delta_{p} \boldsymbol{J}_{i j}^{T}\right]_{k}\right]_{\boldsymbol{q}_{\boldsymbol{i}}}+\left[\left[\Delta_{p} \boldsymbol{J}_{i j}^{T}\right]_{k}\right]_{\boldsymbol{p}_{C i j}} \\
& =\frac{\partial\left[\boldsymbol{J}_{i j}^{T}\right]_{k}}{\partial \boldsymbol{q}_{i}} \quad{ }_{p} \boldsymbol{q}_{\boldsymbol{i}}+\frac{\partial\left[\boldsymbol{J}_{i j}^{T}\right]_{k}}{\partial \boldsymbol{p}_{C i j}^{\boldsymbol{F}}} \quad{ }_{p} \boldsymbol{p}_{C i j}^{F}
\end{aligned}
$$

where $\Delta_{\mathrm{p}} \boldsymbol{q}_{\mathrm{i}}$ denotes vector of joint angle deviation for finger $i^{\text {th }}\left(\Delta_{\mathrm{p}} \boldsymbol{q}_{\mathrm{i}}=\operatorname{col}\left[\Delta_{\mathrm{p}} q_{\mathrm{ij}}\right)\right.$.

The first term indicates the deviation due to the changing of the joint angles without any change in $\boldsymbol{p}^{F}{ }_{i j}$, while the other term indicates the deviation due to the contact point $C_{\mathrm{ij}}$ on the fingertip without any change in the joint angles. Note that the contact position uncertainty causes deviation on both joint angles and contact position on fingertips, as shown in Fig. 2. We consider the two components of the Jacobian deviation separately, as in (22).

The joint angle deviation consists of two factors: contact deviation and low resolution. As assumed in section II, we ignore the deviation due to the resolution. Thus, from (8), the joint angle deviation can be expressed by

$$
{ }_{p} \boldsymbol{q}_{i}=\boldsymbol{J}_{i j}^{+}{ }_{p} \boldsymbol{p}_{C i j}^{o} .
$$

Then $\left[\left[\boldsymbol{J}_{i j}^{T}\right]_{k}\right]_{\boldsymbol{q}_{\boldsymbol{i}}}$ is bounded as follows

$$
\left.\mid\left[\boldsymbol{J}_{i j}^{T}\right]_{k}\right]_{\boldsymbol{q}_{\boldsymbol{i}}}|\leq| \frac{\partial\left[\boldsymbol{J}_{i j}^{T}\right]_{k}}{\partial \boldsymbol{q}_{\boldsymbol{i}}} \boldsymbol{J}_{i j}^{+} \mid P_{\max }^{o} .
$$

Next, we show that the second term of (22), $\left[\boldsymbol{J}_{\mathrm{ij}}{ }^{T}\right]_{\mathrm{k}}$, can be expressed by

$$
\begin{aligned}
{\left[\boldsymbol{J}_{i j}^{T}\right]_{k} } & =\left[\left[\boldsymbol{z}_{i K} \times\right]\left(\boldsymbol{p}_{C_{i j}}^{F}-\boldsymbol{p}_{F i K}\right)\right]^{T}(\mathrm{FPC}) \\
{\left[\boldsymbol{J}_{i j}^{T}\right]_{k} } & =\left[\begin{array}{c}
\left.\left[\boldsymbol{z}_{i K} \times\right]\left(\boldsymbol{p}_{C_{i j}}^{F}-\boldsymbol{p}_{F i K}\right)\right]^{T} \\
\boldsymbol{z}_{i K}
\end{array}\right]^{(\mathrm{soft})}
\end{aligned}
$$

where $\boldsymbol{p}_{\mathrm{FiK}}$ denotes position of proximal joint for $K^{\text {th }}$ link where $C_{\mathrm{ij}}$ exists, and $z_{i K}$ denotes rotation axis of $F_{\mathrm{i} K}$ frame 
[15], as shown in Fig 2. Since we are considering the case when there is no $\Delta_{\mathrm{p}} \boldsymbol{q}_{\mathrm{i}},(24)$ becomes

$$
\begin{aligned}
& {\left[\left[\Delta_{p} \boldsymbol{J}_{i j}^{T}\right]_{k}\right]_{\boldsymbol{p}_{C i j}^{F}}=\left[\left[\boldsymbol{z}_{i K} \times\right] \Delta_{p} \boldsymbol{p}_{C_{i j}}^{F}\right]^{T}(\mathrm{FPC})} \\
& {\left[\left[\Delta_{p} \boldsymbol{J}_{i j}^{T}\right]_{k}\right]_{\boldsymbol{p}_{C i j}^{F}}=\left[\begin{array}{c}
\left.\left[\boldsymbol{z}_{i K} \times\right] \Delta_{p} \boldsymbol{p}_{C_{i j}}^{F}\right]^{T}(\mathrm{soft}) \\
\mathbf{0}
\end{array}\right.}
\end{aligned}
$$

Then we get

$$
\left|\left[\left[\Delta_{p} \boldsymbol{J}_{i j}^{T}\right]_{k}\right]_{\boldsymbol{p}_{C i j}^{f}}\right| \leq\left|\Delta \boldsymbol{p}_{C_{i j}}^{F}\right|=P_{\max }^{F} .
$$

By combining (23) and (25), we can define the maximum Jacobian deviation due to contact position uncertainty as

$$
\left|\left[\Delta_{p} \boldsymbol{J}_{i j}^{T}\right]_{k}\right| \leq\left|\frac{\partial\left[\boldsymbol{J}_{i j}^{T}\right]_{k}}{\partial \boldsymbol{q}_{\boldsymbol{i}}} \boldsymbol{J}_{i j}^{+}\right| P_{\max }^{o}+P_{\max }^{F}={ }_{p} \boldsymbol{J}_{i j k_{\max }}
$$

The magnitude of the contact wrench is bounded as follows

$$
\left|\boldsymbol{w}_{c i j}\right| \leq \sqrt{\mu_{M A X i j}^{2}+1} n_{w_{i j}}
$$

where $\mu_{\mathrm{MAX}}=\max \left(\mu_{\mathrm{sij}}, \mu_{\mathrm{mij}}\right)$. We here leave the proof of (28) as an exercise for you, dear reader.

From (19), (22), (26), and (27) the uncertainty of the $k^{\text {th }}$ joint torque of the $i^{\text {th }}$ finger resulting from the deviation of the $i^{\text {th }}$ finger can be expressed by

$$
\begin{aligned}
&\left|\Delta_{p} \tau_{i k}\right| \leq \sum_{j}\left(\Delta_{p} \tau_{i j k_{\max 1}} \boldsymbol{n}_{i j}^{T} \mathbf{\Xi}_{i j}+{ }_{p} \tau_{i j k_{\max 2}} \boldsymbol{n}_{\boldsymbol{\mu}}^{\boldsymbol{T}}\right) \boldsymbol{w}_{c}(28) \\
&{ }_{p} \tau_{i j k_{\max 1}}=\sqrt{1+\mu_{M A X i j}^{2}}{ }_{p} \boldsymbol{J}_{i j k_{\max }}, \\
&{ }_{p} \tau_{i j k_{\max 2}}=\left|\left[\boldsymbol{J}_{i j}^{T}\right]_{k}\right| \Delta_{p} w_{c i j_{\max } .}
\end{aligned}
$$

Equation (28) shows that when contact uncertainties occur, they act as an additional load on each robot joint. We have to subtract them from the maximum torques supplied by the actuator. As a result, the torque limitation constraint in optimization equation (6) will change such that the required torque, which includes the effect from contact uncertainties, will be lower than the maximum torque.

\section{F. Grasp optimization}

Consider optimization equation (6). From the above derivation, the contact uncertainties will act as additional load on joint torque and affect the frictional condition. Therefore, we adjust the optimization constraint by using (20) as the frictional constraint and substitute (28) into the joint torque limitation. If we assign the task function as the ratio of the required and the available torque and then solve for the internal contact wrenches, we get the linear optimization equation $\operatorname{argmin}_{\gamma} \quad \rho$

subject to

1) $\rho \leq 1$

2) $\left|\tau_{i k}\right| \leq \rho\left|\tau_{i k_{\max }}\right|-\left|\Delta_{p} \tau_{i k}\right|$

3) $\boldsymbol{\tau}=\boldsymbol{J}^{T} \boldsymbol{w}_{c}$

4) $-\boldsymbol{V}_{\boldsymbol{e q}}\left(\boldsymbol{I}-\operatorname{col}\left[\Delta_{p} w_{c i j_{\max }}\right] \boldsymbol{n} \boldsymbol{n}_{\boldsymbol{\mu}}^{T}\right) \boldsymbol{w}_{c} \leq \mathbf{0}$

5) $\boldsymbol{n}_{i j}^{T} \boldsymbol{\Xi}_{i j} \boldsymbol{w}_{c} \geq 0$

6) $\boldsymbol{w}_{c}=-\boldsymbol{G}^{+} \boldsymbol{w}_{\boldsymbol{v}}+\boldsymbol{E} \boldsymbol{\gamma}$

$\left(j=1,2, \ldots, M_{\mathrm{i}}, i=1,2, \ldots, N, \mathrm{l}=1,2, \ldots, n_{\mathrm{rew}}\right)$

where $\left|\tau_{i k \max }\right|$ is maximum value of $\left|\boldsymbol{\tau}_{i k}\right|$. If the required joint torque is above this value, we conclude that we cannot grasp the object. In this algorithm, we try to find the internal contact wrench $\gamma$ that produces the minimum $\rho$ under the derived grasping conditions (where $\rho$ denotes the ratio between the required torque and the maximum torque). Conditions 1, 2, and 3 indicate joint torque limitations: conditions 4 and 5 indicate frictional conditions: and condition 6 indicates internal and external contact wrenches.

If linear programming equation (29) cannot be solved, it means that the probability that at least one of the constraints cannot be satisfied is high. The grasp posture is not good for supporting defined uncertainty. Therefore a new grasp posture should be considered. If we can obtain a solution $\gamma=$ $\boldsymbol{\gamma}^{*}$, we will derive the corresponding desired joint angle $\boldsymbol{q}_{\mathrm{d}}=$ $\left[q_{\mathrm{d} 11} q_{\mathrm{d} 12} \ldots\right]^{\mathrm{T}}$ using the following relationship:

$$
\boldsymbol{q}_{\mathrm{d}}=\boldsymbol{K}^{\mathbf{1}} \boldsymbol{J}^{\mathbf{T}} \boldsymbol{E}^{*}+\boldsymbol{q}_{\text {nominal }}
$$

After obtaining the desired hand posture, we can find the necessary torque based on compliance controller mentioned in section II. This grasp input (joint torque input corresponding to $\gamma^{*}$ ) can generate the required internal contact wrenches.

\section{SIMULATION AND EXPERIMENTAL RESULTS}

In this paper, we use examples to verify our approach. We used the robot hand called "Kanazawa Hand" [16], developed in our laboratory. This hand has 4 fingers and 12 degree of freedom (DOFs). The selected target object was a $3.8 \mathrm{~cm}$ radius, $2.5 \mathrm{~cm}$ high cylinder. The designed contact points were as depicted in Fig. 5.

This robot grasps objects from the $+y$ direction, as illustrated in Fig. 5. The simulation conditions are shown in Table I. The frictional constraint was represented by an octahedral convex cone. The gravitational direction was $-\mathbf{y}$. The object's frame was set at origin $(0,0,0)$. For simplicity, we assumed that $\left(P_{\max }^{F}\right)$ was equal to $\left(P_{\max }^{O}\right)$, and they were represented as $P_{\max }$, in this simulation.

TABLE I

SIMULATION CONDITION

\begin{tabular}{|l|l|}
\hline Coefficient of friction $\left(\mu_{\mathrm{s}} \& \mu_{\mathrm{m}}\right)$ & $0.3 \& 0.1$ \\
\hline Nominal CM position & $(0,0,0)$ \\
\hline Maximum torque & 0.805 N.m (for proximal joints) \\
\hline Maximum torque & 0.232 N.m (for the other joints) \\
\hline
\end{tabular}




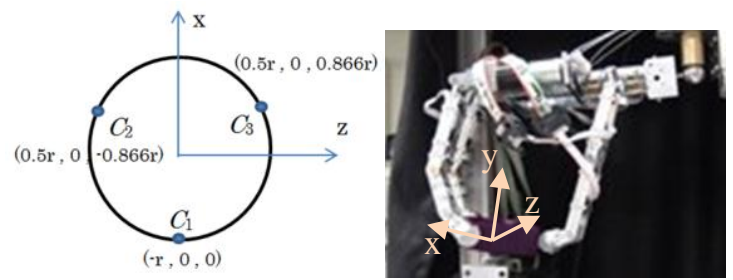

Fig 5 Contact position and used grasp posture with Kanazawa Hand

\section{A. Allowable deviation of $C M$}

First, we looked at the effect of contact uncertainty on graspable load and the deviation of center of mass (CM). Normally, we cannot accurately estimate the position of CM for general objects and this causes the grasping system to become more burdened. This deviation affects the moment component of the external wrench, which is equal to the cross product of its force component and the CM deviation. Therefore, we set $\boldsymbol{w}_{\mathrm{vi}}$ for REWS as follows:

$$
\begin{aligned}
& n_{\text {rew }}=5, \boldsymbol{w}_{\mathrm{vl}}=[0,0,0,0,0,0] \\
& \boldsymbol{w}_{\mathrm{v}(1+2 \varepsilon 1+\varepsilon 2)}=w_{\max }\left[0,-1,0, P_{\mathrm{CMmax}}(-1)^{\varepsilon l}, 0, P_{\mathrm{CM} \max }(-1)^{\varepsilon 2}\right] \text {, } \\
& \varepsilon 1, \varepsilon 2 \in\{1,2\}
\end{aligned}
$$

where $w_{\max }$ represents maximum supportable object's load and $P_{\mathrm{CMmax}}$ represents the maximum CM deviation. Since the only external force is in the y-direction, the external moment due to $\mathrm{CM}$ deviation in the y-direction was set to zero.

We also considered the relation between the graspable load and the deviation of CM at one constant $P_{\max }$. We investigated this relation by choosing $P_{\max }$ and $\boldsymbol{w}_{\max }$ then finding the maximum $P_{\mathrm{CMmax}}$ that could be achieved by (29). Then we varied $\boldsymbol{w}_{\max }$. In this simulation we considered the cases when $P_{\max }=0,1,2$, and $3 \mathrm{~mm}$. The simulation results are shown in Fig. 6.
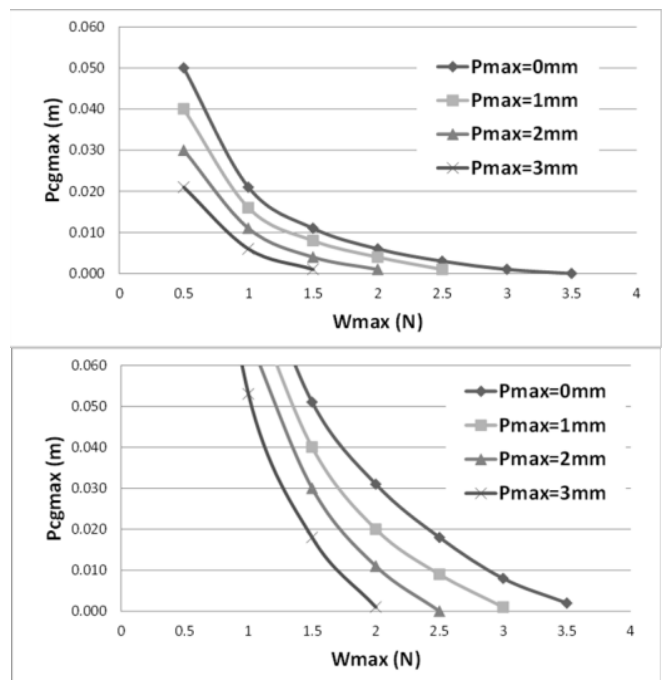

Fig 6 Relations between maximum supportable object's load and maximum CM deviation under different maximum contact uncertainties (above; FPC, below; Soft finger contact)

From the simulation results, it can be seen that at a constant $P_{\max }$ the supportable load decreases when the CM deviation increases. When $P_{\max } \neq 0$, the grasping ability of robot decreased. In other words, when $P_{\max }$ increases, more accuracy in CM position estimation is required for supporting the same load. When $P_{\max }$ increases, the robot hand has to grasp a lighter load at the same accuracy of CM estimation.

The figure also indicates the maximum contact uncertainty that the grasp system can support at the specific load and accuracy of $\mathrm{CM}$ estimation. For example, suppose that the robot hand grasps the $2 \mathrm{~N}$ object with perfect $\mathrm{CM}$ estimation. The maximum contact uncertainty for this system would be 2 $\mathrm{mm}$ (if we consider FPC). However, if the contact uncertainty is higher than $2 \mathrm{~mm}$, the robot cannot grasp the object at this setting.

When FPC and soft finger contact are compared, we see that, in contrast to FPC, soft finger contact can support more uncertainty for both $\mathrm{CM}$ deviation and contact uncertainty.

\section{B. Experimental results}

In this section, we show the validity of our approach (29) and compare it to conventional approaches (6). We applied our algorithm to a real robot. The experiment was set up as illustrated in Fig. 5. The experimental conditions set were as follows. The weight of the object is $1 \mathrm{~N}$. A contact uncertainty of $3 \mathrm{~mm}$ was assumed, and the CM position was precisely obtained. From linear programming equation (29), we obtained the internal contact wrench $\boldsymbol{E} \gamma$ as in Table II. Note that, we can obtain the joint torque from both (1) and (30), but we show the internal contact wrench, which is easier to

\begin{tabular}{|c|c|c|c|c|c|}
\hline \multirow[b]{2}{*}{ point } & & \multicolumn{2}{|c|}{ FPC } & \multicolumn{2}{|c|}{ Soft finger } \\
\hline & & proposed & normal & proposed & normal \\
\hline \multirow[t]{4}{*}{$C_{1}$} & $f_{\mathrm{x}}$ & 3.127 & 1.58 & 2.53 & 1.564 \\
\hline & $f_{\mathrm{y}}$ & 0 & 0 & -0.136 & -0.13 \\
\hline & $f_{\mathrm{z}}$ & 0 & -0.09 & 0.535 & -0.013 \\
\hline & $\phi$ & & & 0 & 0 \\
\hline \multirow[t]{4}{*}{$C_{2}$} & $f_{\mathrm{x}}$ & -1.563 & -0.907 & -1.166 & -0.805 \\
\hline & $f_{\mathrm{y}}$ & 0 & 0 & 0.078 & 0.055 \\
\hline & $f_{\mathrm{z}}$ & 0.9027 & 0.456 & 0.673 & 0.465 \\
\hline & $\phi$ & & & -0.01 & -0.009 \\
\hline \multirow[t]{4}{*}{$C_{3}$} & $f_{\mathrm{x}}$ & -1.5634 & -0.774 & -1.365 & -0.758 \\
\hline & $f_{\mathrm{y}}$ & 0 & 0 & 0.058 & 0.075 \\
\hline & $f_{\mathrm{z}}$ & -0.9027 & -0.4466 & -0.726 & -0.452 \\
\hline & $\phi$ & & & 0.01 & 0.009 \\
\hline \multicolumn{2}{|c|}{$\rho$} & 0.59 & 0.275 & 0.486 & 0.271 \\
\hline
\end{tabular}
understand.

TABLE II

The unit for force component is Newton, and for moment component is Newton-meter.

It can be seen that there is an approximate twofold increase in the required contact wrench when we consider contact uncertainty for this grasping condition. In addition, the required contact wrenches are lower when we use soft finger contact as the frictional constraint. We applied grasp input to the robot so that the robot could generate the contact wrench in Table II, and we verified the result. In this experiment, the result was categorized into four groups (A-D), as shown in Fig. 7. The explanation of each group is as follows.

Group A: The robot successfully grasped and lifted the object and the object had the same orientation as it did before it was grasped. 
Group B: The robot successfully grasped and lifted the object but the object tilted due to slippage.

Group C: The robot successfully grasped and lifted the object but the object fell after a period of time had elapsed.

Group D: The robot was not able to lift the object.



Fig 7 The number of grasping results categorized into each group

From the experimental results, it can be seen that the probability of successful grasping was higher when we used our proposed grasp optimization algorithm. The robot was, for the most part, able to grasp the object successfully (groups A and B), while with the conventional grasp optimization technique, the robot could hardly grasp the object. However, in this experiment, our proposed grasp optimization technique still could not guarantee a $100 \%$ success rate and the results were not consistent for all 10 times the experiment was conducted. A possible reason for this may be attributed to factor affecting the performance of the robot hand such as backlash and static friction of the robot. Static friction means that there is a threshold for robot movement. When there is static friction, the robot does not response if the input torque is smaller than the threshold. Moreover, when there is backlash in the system, we cannot guarantee that the robot can move to the required posture, $\boldsymbol{q}_{\boldsymbol{d}}$, even when it receives the desired position from the encoder. The backlash can be compensated for but the exact range of the backlash varies and is very hard to specify.

The results show that the chance of successful grasping when using soft finger contact is a little bit lower than that of FPC. However, it does not mean that the robot can grasp better with FPC. The reason is that theoretically, if we use soft finger contact analysis, the grasp system requires less contact wrenches to support the same external wrench. In other words, the results show that the possibility of a successful grasp increases if the internal contact wrenches increase. Note that we used the same experimental setup while the contact model changed.

\section{CONCLUSION}

This paper presented new grasp optimization technique pertaining to contact position and object information uncertainties. In section III, we reviewed the basic formula necessary for a grasping system. In section IV, we first verified the boundary of contact uncertainties and its effect on normal contact direction (if any). We then verified the effect of contact uncertainty on each grasp constraint (i.e., grasp equation, frictional condition, and joint torque limitation) and its boundary. Finally, we summarized all of the affected constraints into linear grasp optimization. In this paper, we optimized the applied joint torque for solving internal contact wrenches. We included the external wrench uncertainties in REWS and applied REWS to linear grasp optimization instead of one exact external wrench. Numerical solutions and experiments were then conducted to verify the validity of our approach. We found that, theoretically, increasing the internal contact wrenches can compensate for small contact uncertainties. The size of the uncertainties that can be compensated for vary for grasp posture, size of external wrench, and available torques. By considering torque limitation and contact position uncertainty, we evaluated whether a robot hand can grasp an object successfully, with greater accuracy. In the future, the imperfections in the robot hand will be taken in to account so that grasp optimization can be guaranteed even when contact uncertainty occurs.

\section{REFERENCES}

[1] K. B. Shimoga, "Robot grasp synthesis algorithms: A survey," Int. J. Robot. Res., vol. 15 no.3, 1996, pp.230-266.

[2] A. Bicchi and V. Kumar, "Robotic grasping and contact: A review," IEEE Intl. Conf. on Robotics and Automation, 2000, pp.348-353.

[3] J.R. Kerr and B. Roth, "Analysis of multi-fingered hands," International Journal of Robotics Research 4(4), 1986.

[4] T.Watanabe and T. Yoshikawa, "Grasping optimization using a required external force set," IEEE Transactions on Automation Science and Engineering vol. 4, no1, 2007, pp. 52-66.

[5] C.C. Cheah, H.Y. Han, S. Sawamura, and S. Arimoto, "Grasping and position control for multi-fingered robot hands with uncertain Jacobian matices," Proceedings of the IEEE International Conference on Robotics and Automation (ICRA), 1998, pp. 2403-2408.

[6] T.Schlegl and M. Buss,"Hybrid closed-loop control of robotic hand regrasping," Proceeding of the IEEE International Conference on Robotics and Automation (ICRA), 1998, pp. 3026-3031.

[7] B.M. Bone and Y. Du, "Multi-Metric Comparison of optimal 2D grasp planning algorithms," in Proceedings of the IEEE international conference on robotics and automation, vol. 3, Seoul, Korea, 2001, pp. 3061-3066

[8] Y.Zheng, W. Qian "Coping with the grasping uncertainties in Force-closure analysis," The international journal of robotics research, vol. 24, no.4, 2005, pp. 311-327

[9] V.Christopoulosand, P. Schrater, "Handling shape and contact location uncertainty in grasping two-dimensional planar objects," in Intelligent Robots and Systems, 2007. IROS 2007. IEEE/RSJ International Conference, pp. 1557-1563

[10] J.Glover, D.Rus, and N.Roy, "Probabilistic models of object geometry with application to grasping," International journal of robotics research, v. 28(8), 2009

[11] D.Berenson, S.Srinivasa, and J. Kuffner, "Addressing pose uncertainty in manipulator planning using task space regions," Intelligent Robots and Systems, 2009. IROS 2009

[12] P. Fungtammasan, and T. Watanabe, "Grasp input optimization taking contact position uncertainty into consideration," Proceeding of IEEE-ROBIO 2011

[13] I.Kao, and M.R. Cutkosky, "Quasistatic manipulation with compliance and sliding," International journal of robotics research, 1992, vol.11, no.1, pp.20-40.

[14] Z. Yu, and Q. Wenhan, "Linearizing the soft finger contact constraint with application to dynamic force distribution in multifingered grasping", Science in China Engineering \& Material Science vol48 no.2, 2005,pp 121-130.

[15] M.W. Spong, S. Hutchinson, and M. Vidyasqar, "Robot modeling and control," ISBN 978-0-471-64990-8, 2005

[16] H. Takeuchi, and T Watanabe, "Development of a multi-fingered robot hand with softness - changeable skin mechanism," Proceeding of the joint conference of ISR 2010 and ROBOTIK 2010 